\title{
REALIZING SYMMETRIES OF A SUBSHIFT OF FINITE TYPE BY HOMEOMORPHISMS OF SPHERES
}

\author{
BY J. B. WAGONER ${ }^{1}$
}

Let $A$ be a finite, irreducible, zero-one matrix and let $\sigma_{A}: X_{A} \rightarrow X_{A}$ be the corresponding subshift of finite type $[\mathbf{F}]$. Recall from $[\mathbf{F}]$ that a Smale diffeomorphism is one with a hyperbolic zero-dimensional chain recurrent set. A well-known theorem of Williams-Smale [Wi] says that there is a Smale diffeomorphism $F_{A}: S^{3} \rightarrow S^{3}$ so that $\sigma_{A}$ is topologically conjugate to the restriction of $F_{A}$ to the basic set of index one occurring as part of the spectral decomposition. Let $\operatorname{Aut}\left(\sigma_{A}\right)$ denote the group of symmetries of $\sigma_{A}$-that is, the group of homeomorphisms of $X_{A}$ which commute with $\sigma_{A}$. Here is the corresponding global realization result for these symmetries.

THEOREM. Assume $4<q$ and let $1<e<q-2$. Then there is a Smale diffeomorphism $F_{A}: S^{q} \rightarrow S^{q}$ with a basic set $\Omega_{e}$ of index $e$ (along with other basic sets of index $0, e+1, q)$ together with a topological conjugacy between $\sigma_{A}$ and $F_{A} \mid \Omega_{e}$ so that, given any symmetry $g$ in $\operatorname{Aut}\left(\sigma_{A}\right)$, there is a homeomorphism $G: S^{q} \rightarrow S^{q}$ satisfying

(A) $G$ commutes with $F_{A}$ on all of $S^{q}$,

(B) $G \mid \Omega_{e}=g$ under the identification between $\operatorname{Aut}\left(F_{A} \mid \Omega_{e}\right)$ and $\operatorname{Aut}\left(\sigma_{A}\right)$.

The motivation and the idea for the proof of this geometric result came by analogy from algebraic $K$-theory and pseudo-isotopy theory. The proof uses Williams' notion of strong shift equivalence $[\mathbf{W} \mathbf{1}, \mathbf{F}]$, the contractible simplicial complex $P_{A}$ of topological Markov partitions for $\sigma_{A}[\mathbf{W 1}$, and structural stability for Smale diffeomorphisms $[\mathbf{R}, \mathbf{R o}]$. We would like to thank C. Pugh for useful discussions about the stability theorem.

The group $\operatorname{Aut}\left(\sigma_{A}\right)$ is often rather large. For example, Aut $\left(\sigma_{2}\right)$ for the Bernoulli 2-shift $\sigma_{2}$ has been known $[\mathbf{H}]$ for some time to contain every finite group and to have elements of infinite order not a power of $\sigma_{2}$. Recently, Boyle and Lind have shown it contains the free nonabelian group on infinitely many generators. Therefore, the group of homeomorphisms of $S^{q}$ commuting with a certain $F_{2}$ is large when $4<q$. Incidentally, at the present time not much is really known about the structure and other algebraic or homological properties of $\operatorname{Aut}\left(\sigma_{2}\right)$. For some information see [BK] or [W1]. An open and long-standing conjecture is that $\operatorname{Aut}\left(\sigma_{2}\right)$ is generated by $\sigma_{2}$ and elements of finite order.

Here is a rough idea of the proof of the Theorem. The details will appear in [W2]. Let $P$ be an $m \times m$ zero-one matrix and let $Q$ be an $n \times n$ zero-one matrix. Suppose there is an $m \times n$ zero-one matrix $R$ and an $n \times m$ zero-one

Received by the editors September 30, 1985.

1980 Mathematics Subject Classification (1985 Revision). Primary 34C35, $20 \mathrm{~B} 27$.

${ }^{1}$ Partially supported by the NSF. 
matrix $S$ so that $P=R S$ and $Q=S R$. As in [Wi], this determines a specific conjugacy $c_{R}:\left(X_{P}, \sigma_{P}\right) \rightarrow\left(X_{Q}, \sigma_{Q}\right)$ sending $x=\left\{x_{i}\right\}$ in $X_{P}$ to $c_{R}(x)=$ $\left\{c_{R}(x)_{i}\right\}$ in $X_{Q}$, where $c_{R}(x)_{i}$ is the unique $k$ such that $R\left(x_{i}, k\right) S\left(k, x_{i+1}\right)=$ $A\left(x_{i}, x_{i+1}\right)=1$. Similarly for $c_{S}$. In fact, $c_{S} c_{R}=\sigma_{P}$ and $c_{R} c_{S}=\sigma_{Q}$, so that $c_{R} \sigma_{P}=\sigma_{Q} c_{R}$ and $c_{S} \sigma_{Q}=\sigma_{P} c_{S}$. We call $c_{R}$ and $c_{S}$ elementary symbolic conjugacies.

On the topological side, let $S^{q}(m)$ be the standard $q$-sphere equipped with a fixed handle decomposition with one handle of index zero, $m$ handles of index $e, m$ cancelling handles of index $e+1$, and one handle of index $q$. Similarly for $S^{q}(n)$. One then constructs a Smale diffeomorphism $C_{R}: S^{q}(m) \rightarrow S^{q}(n)$ which is fitted both on the handles of index $e$ and the handles of index $e+1$ according to the geometric intersection matrix $R$. Again, similarly for $C_{S}$. This is done in such a way that the composition $D_{P}=C_{S} C_{R}: S^{q}(m) \rightarrow S^{q}(m)$ is also a Smale diffeomorphism fitted on the $e$-handles and $(e+1)$-handles according to the matrix $P=R S$ and $D_{Q}=C_{R} C_{S}: S^{q}(n) \rightarrow S^{q}(n)$ is fitted according to $Q=S R$. Observe that $C_{R} D_{P}=D_{Q} C_{R}$ and $D_{P} C_{S}=C_{S} D_{Q}$, and therefore $C_{R}$ and $C_{S}$ are smooth conjugacies between $D_{P}$ and $D_{Q}$. We call these elementary smooth conjugacies.

Now consider a Smale diffeomorphism $F_{P}: S^{q}(m) \rightarrow S^{q}(m)$ which is fitted on the $e$-handles and $(e+1)$-handles by the matrix $P$. In general, of course, $F_{P} \neq D_{P}$. However, under the assumption that $1<e<q-2$ we are able to carefully construct $F_{P}, C_{R}$, and $C_{S}$ in such a way that there is a oneparameter family of Smale diffeomorphisms $F_{P}(t)$, each of which is fitted on the $e$-handles and $(e+1)$-handles by the matrix $P$, so that $F_{P}(0)=F_{P}, F_{P}(1)$ is equal to $D_{P}$ on a neighborhood of the $(e+1)$-skeleton, and both $F_{P}(1)$ and $D_{P}$ have the point at infinity as a source. Methods of stability theory $[\mathbf{R}, \mathbf{R o}]$ can then be used to produce a topological (not smooth) conjugacy between $F_{P}$ and $D_{P}$. We call this a stability conjugacy. Similarly, there is a stability conjugacy between $D_{Q}$ and $F_{Q}$, so that we then get a topological conjugacy $F_{P}$ and $F_{Q}$.

The main theorem is proved by first showing that any symmetry $g$ in $\operatorname{Aut}\left(\sigma_{A}\right)$ can be obtained as the composition of a chain of elementary symbolic conjugacies and powers of shifts, and then by showing this can be mirrored compatibly with a corresponding chain of elementary smooth conjugacies, stability conjugacies, and powers of certain intermediate $F_{P}$ for different matrices $P$. The chain starts with the original $F_{A}$ which is fixed and eventually comes back to it. The composition of the various conjugacies and powers of $F_{P}$ in the chain give the required homeomorphism $G$.

The main theorem may well be valid on $S^{4}$ also, but our argument seems to require $4<q$.

\section{REFERENCES}

[BK] M. Boyle and W. Krieger, Periodic points and automorphisms of the shift, preprint, Univ. of Maryland/Inst. Angew. Math. der Univ. Heidelberg, 1985.

[F] J. Franks, Homology and dynamical systems, CBMS Regional Conf. Ser. in Math., no. 49, Amer. Math. Soc., Providence, R.I., 1982. 
[H] G. Hedlund, Endomorphisms and automorphisms of the shift dynamical system, Math. Systems Theory 3 (1969), 320-375.

[R] J. Robbin, A structural stability theory, Ann. of Math. (2) 94 (1971), 447-493.

[Ro] C. Robinson, Structural stability for $C^{1}$ diffeomorphisms, J. Differential Equations 22 (1976), 28-73.

[W1] J. B. Wagoner, Markov partitions and $K_{2}$, preprint, Univ. of California, Berkeley, 1985.

[W2] _ _ Realizing symmetries of a shift, preprint, Univ. of California, Berkeley, 1985.

[Wi] R. F. Williams, Classification of subshifts of finite type, Ann. of Math. (2) 98 (1973), 120-153; errata 99 (1974), 380-381.

Department of Mathematics, University of California, Berkeley, CALIFORNIA 94720 
